\title{
EDITORIAL
}

\section{Large-Scale Assays}

\section{Elias Ayres Guidetti Zagatto (D) $\triangle$}

Full Professor

Centre for Nuclear Energy in Agriculture, University of Sao Paulo

Piracicaba, SP, Brazil

A decade ago (June $18^{\text {th }}$ - the Chemist Day in Brazil), BrJAC was launched as "the seed of our dream of fostering integration between academia, research centers, and industry at this time of growth for graduate programs at the international level" [1].

As the first Brazilian scientific journal entirely dedicated to Analytical Chemistry, it underwent a remarkable development, as demonstrated by the continuous increase in e.g. impact factor, widespread dissemination, and recognition by the scientific community. This reflects the quality of the scientific articles, reviews and technical notes, as well as opinions of the community through editorials, points of view, letters, interviews, sponsor reports and releases. The BrJAC involvement in scientific meetings, the "Young Talent in Analytical Chemistry Award", the enthusiastic collaboration of the analytical community, and the logistic of manuscript handling, as well as the dedication of the editing staff should also be highlighted.

Regarding enhancement of the scientific interaction between universities, research institutes and companies, an important aspect refers to the management of laboratories dedicated to large-scale analysis, as testified by me at the Centre for Nuclear Energy in Agriculture (CENA), University of Sao Paulo during the seventies. As a member of CENA's analytical team, I experienced an important moment in the context of Analytical Chemistry: the development of a novel concept of automated chemical analysis - flow injection analysis - and its pioneer exploitation for large-scale assays [2,3].

The innovation led to an outstanding increase in the productivity of the laboratory, allowing analysis relevant to all CENA projects to be performed in a very short time, with a low cost-benefit ratio, and excellent figures of analytical merit. Other Institutions and private initiatives started to request analysis, thus establishing a healthy situation combining research development and services.

Analysis of atypical samples with unexpected matrix composition, concentrations of potential interfering species and/or out-of-range analyte concentrations required other specific strategies. This was the driving force towards additional developments in flow analysis, providing themes for more realistic and relevant research. This aspect culminated with the proposal of expert flow analyzers, which enable the real-time implementation of flow and manifold modifications. Consequently, the research quality, idea dissemination, and integration of people with different formations have been improved.

Nowadays, it is recommended that, regardless of the involved analytical instruments, an academic or an industrial laboratory for large-scale analysis should contemplate two goals: performing repetitive assays of typical samples, and carrying out research to solve difficulties that are inherent to atypical samples. All individuals involved should be able to work in both situations. This results in a natural establishment of research teams, an increased university-enterprise relationship, a more realistic and relevant research, and improvements in the laboratory capacity, among others. Last, but not least, the quality of the analytical results, the originality, relevance and suitability of the research, and the importance of the related scientific papers will certainly be better.

Cite: Zagatto, E. A. G. Large-Scale Assays. Braz. J. Anal. Chem., 2020, 7 (27), pp 1-2. doi: http://dx.doi.org/10.30744/ brjac.2179-3425.editorial.ezagatto.N27 
The role of BrJAC in the near future is therefore to continue supporting analytical chemistry, both on the academic and industrial side. The quality of each published issue should always be higher than that of the previous one. Enjoy reading this issue.

\section{REFERENCES}

1. Kubota, L. T. Braz. J. Anal. Chem., 2010, 1 (1) pp V-V.

2. Hansen, E. H.; Ruzicka J. TrAC, Trends Anal. Chem., 1983, 2 (7) pp V-VII.

3. Cerqueira, A. Braz. J. Anal. Chem., 2010, 1 (1) pp XIV-XVII.

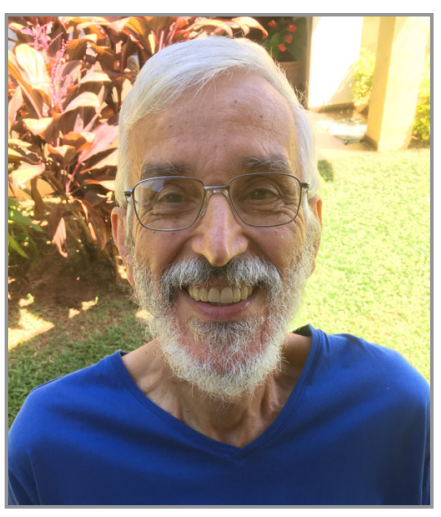

Elias Ayres Guidetti Zagatto has a degree in Agronomic Engineering from the University of São Paulo (1971), a master degree in Nuclear Energy in Agriculture from the University of São Paulo (1974) and a doctoral degree in Analytical Chemistry from the University of Campinas (1981). He is currently a Professor at the Center for Nuclear Energy in Agriculture, University of São Paulo, and a Member of the Brazilian Academy of Sciences. His research activities mainly include the design and development of flow analyzers, with applications on relevant samples in the agronomic, environmental, pharmaceutical and industrial areas.

CV: http://lattes.cnpq.br/4073076139407519

$\mathrm{p}$ ResearcherID 